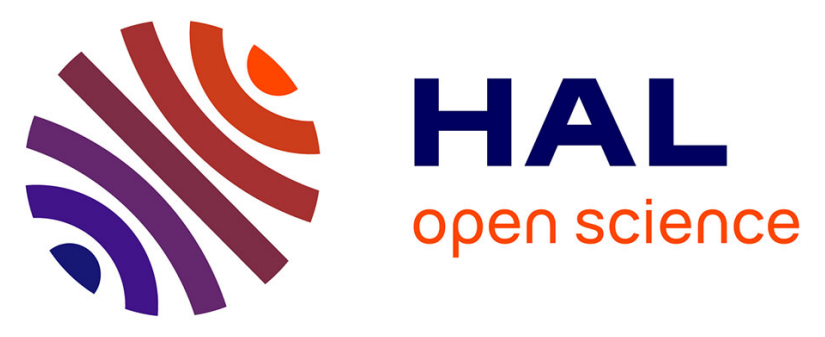

\title{
Design of Supervisory Control System for Ventricular Assist Device
}

\author{
André Cavalheiro, Diolino Santos Fo., Aron Andrade, José Roberto Cardoso, \\ Eduardo Bock, Jeison Fonseca, Paulo E. Miyagi
}

\section{- To cite this version:}

André Cavalheiro, Diolino Santos Fo., Aron Andrade, José Roberto Cardoso, Eduardo Bock, et al.. Design of Supervisory Control System for Ventricular Assist Device. 2nd Doctoral Conference on Computing, Electrical and Industrial Systems (DoCEIS), Feb 2011, Costa de Caparica, Portugal. pp.375-382, 10.1007/978-3-642-19170-1_41 . hal-01566574

\section{HAL Id: hal-01566574 \\ https://hal.inria.fr/hal-01566574}

Submitted on 21 Jul 2017

HAL is a multi-disciplinary open access archive for the deposit and dissemination of scientific research documents, whether they are published or not. The documents may come from teaching and research institutions in France or abroad, or from public or private research centers.
L'archive ouverte pluridisciplinaire HAL, est destinée au dépôt et à la diffusion de documents scientifiques de niveau recherche, publiés ou non, émanant des établissements d'enseignement et de recherche français ou étrangers, des laboratoires publics ou privés. 


\title{
Design of Supervisory Control System for Ventricular Assist Device
}

\author{
André Cavalheiro ${ }^{1}$, Diolino Santos Fo. ${ }^{1}$, Aron Andrade ${ }^{2}$, José Roberto Cardoso ${ }^{1}$, \\ Eduardo Bock ${ }^{2}$, Jaison Fonceca ${ }^{2}$, Paulo Eigi Miyagi ${ }^{1}$ \\ ${ }^{1}$ Department of Mechatronics and Mechanical Systems Engineering, Escola Politécnica da \\ USP, São Paulo, Brazil \\ ${ }^{2}$ Department of Bioengineering, Institute Dante Pazzanese of Cardiology, São Paulo, \\ Brazil
}

\begin{abstract}
When a patient have severe heart diseases, Ventricular Assist Device (VAD) implantation may be necessary. However, the improvement of the interaction between the device and the patient's behavior is crucial. Currently, the control of these pumps does not follow changes in patient behavior and the devices are no safe. Therefore, if VAD has no faults tolerance and no dynamic behavior according to the cardiovascular system performance, there is a serious limitation on expected results. This research investigates a mechatronic approach for this class of devices based on advanced techniques for control, instrumentation and automation to define a method for developing a hierarchical supervisory control system to control a VAD dynamically and securely. To apply this method, concepts based on Petri nets and Safety Instrumented Systems are used. This innovation reduces the interventions and unnecessary drugs, enabling a reduction of deposable material and patient hospitalization, and contributes to sustainability concept..
\end{abstract}

Keywords: Ventricular Assist Device, Petri nets, Safety Instrumented System.

\section{Introduction}

The increase of resources consumption on the world is one of the main features that justify the use of sustainability concept on methods for projects development. In this sense, automation can help to optimize the resources utilization and to improve devices performance. Thus, this work proposes a development of Ventricular Assist Device (VAD) to aid patient with heart failure to be able to have relatively normal life despite the disease applying sustainability concepts. This device can be used in several cases during the period that patient is waiting for heart transplantation, during a pre or postoperative recovery period, or as destination therapy when the patient has no indication for heart transplantation due to several reasons $[1,2,3]$. VAD projects involve many research areas: mechanical and electromechanical engineer, biomaterial, medicine, and also computer technologies for data collection, processing and making decision, therefore, sensors to indicate blood pressure, blood flow, body temperature and cardiac frequency are necessary [4]. Figure 1 represents how the VAD interacts with the cardiovascular system. 


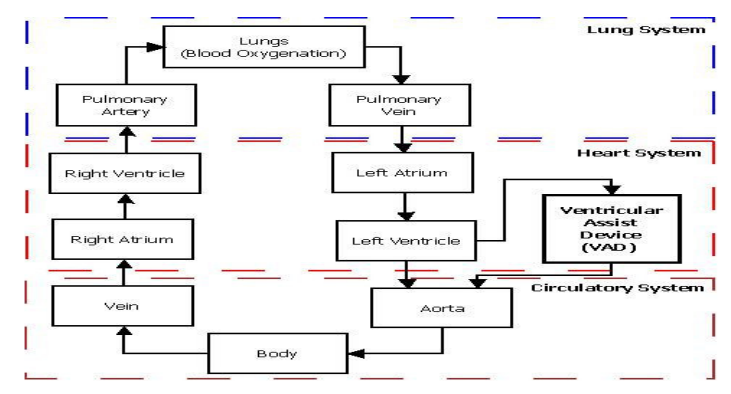

Fig. 1. VAD and cardiovascular system

In this context, two main aspects must be considered:

- First: device shall demonstrate correct and accurate performance; otherwise, if the pump fail during operation and there is no embedded system that enables the treatment of faults autonomously, serious risks to patient will be inevitable.

- Second: many VADs maintain constant blood flow regardless to patient daily needs [5], ie, they help blood circulation and do not react properly to changes [6].

Therefore, if VAD has no faults tolerance and no dynamic behavior according to the cardiovascular system performance serious limitation on results from this application may be observed. This work proposes application of a mechatronic approach based on advanced techniques for control, instrumentation and automation. These techniques allow treatment of fundamental limitations of current solutions. So, we propose a method for specifying a supervisory control system for a VAD that:

- Specify a logic for pump speed control, according to patient's dynamic behavior. Models based on Bayesian networks (BN) [12] should be applied to diagnose patient's dynamic state, at every moment and to act in VAD control.

- Specify a logic for safety interlock to prevent faults in VAD. We must diagnose the critical states by using BN and implement a diagnosis system by using Petri nets (PN). Once implemented the diagnosis control system, in parallel, should be implemented faults treatment according to specification of safety instrumented functions (SIFs) [10, 11]. These functions must be modeled in PN [9] for generating the control algorithm for faults treatment.

- Check the mathematical model of supervisory control system according to its interaction with a model of human cardiovascular system $[16,17,18]$.

So, supervisory control system can be implemented and specified for in vitro and in vivo testing in a consistent way.

\section{Technological Innovation and Sustainability}

There are three conflicting aspects that are considered if a new strategic approach as "Sustainability research" is adopted, i.e., contributing to economic development, being ecologically acceptable and socially just [20]. This approach will guide sustainable solutions that have to be more effective and efficient spending of public 
and private funds for research and development. To do the sustainability and make this a brand for the 21 st century requires a strong engagement of science, industry and politics.

Considering also previous works in the area of VADs, here we adopt the approach of a hybrid system presents dynamical behavior in which simultaneous evolution of continuous and discrete state variables occurs, ie, Continuous Variable Systems (CVS) behavior merge with Discrete Event Systems (DES) behavior [8]. Considering the requirement that a VAD needs to perform control functions to adjust pump speed according to changes on cardiac frequency and needs to react against occurrence of critical faults, we proposed a hybrid supervisory control system [7].

In this context, this research will contribute to add to VAD the following features: rational use of energy, minimization of operational costs and larger safety and comfort to the users. Then, this work is also a contribution for the technological innovation in design methods of VADs.

\section{Materials and Methods}

Production Flow Schema (PFS) is a technique that can be used to model the set of activities that VAD can perform. The PFS is a bipartite graph composed of activity elements (action, execution), distributing elements (collect, accumulate and/or store information or items) and oriented arcs to connect the elements. Figure 2(a) shows the graphical representation of these elements.

Details of each activity modeled in PFS can be refined using PN which are capable of representing dynamic behavior of device. As VAD has continuous variables, Hybrid Petri Nets (HPN) are necessary.

HPN model has been introduced as extension of discrete PN model been able to handle real numbers in continuous way and allowing us to express explicitly the relationship between continuous values and discrete values while keeping good characteristics of discrete PN soundly. In HPN model, two kinds of places and transitions are used: discrete/ continuous places and discrete/continuous transitions. A continuous place holds a nonnegative real number as content. A continuous transition fires continuously in the HPN model and its firing speed is given as function on model places. Figure 2(b) shows graphical notations of HPN elements [9]. The refinement of a model generated in PFS for a model in HPN is made based on the procedure adopted in Villani [7].

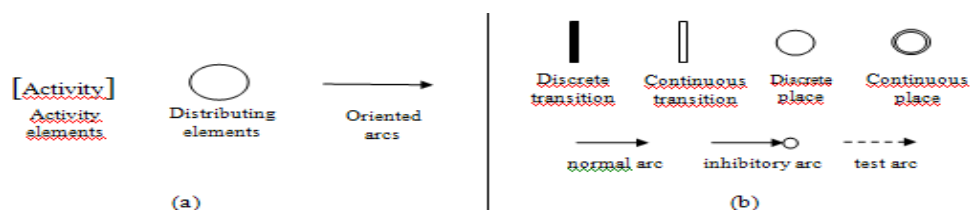

Fig. 2. (a) Production Flow Schema elements; (b) Basic elements of Hybrid Petri Net [9]

For modeling of diagnosis and treatment of critical faults, we are using the concept of Safety Instrumented System (SIS) and BN. According to Squillante [10] SIS is a 
layer of control in order to mitigate the risk or taking the process in a safe state. Definition of faults is made from the identification of Safety Instrumented Functions (SIF). In this way, a SIF describes a critical fault that should be diagnosed and treated by SIS. A SIS implements its SIFs through sensors and devices perform control by actuators. For each SIF a parameter called Safety Integrity Level (SIL) is defined. This parameter is a measurement of safety for components and/or systems. SIL reflects what end users can expect from a device and/or system in a safety function, and in case of fault, this occurs in a safety way. BNs provide formalism for reasoning about partial beliefs under uncertainty conditions. The propositions are as numerical parameters signifying the belief degree according to some evidence or knowledge. So, formally, BNs B = (G; Pr) are made up by a topological structure $G$ and a set of parameters Pr that represents probabilistic relationship among their variables [10, 11].

Therefore, to develop a design for supervisory control system for VAD, according to the modeling techniques presented above, proposes a method for developing the VAD a supervisory system shown in Figure 3:

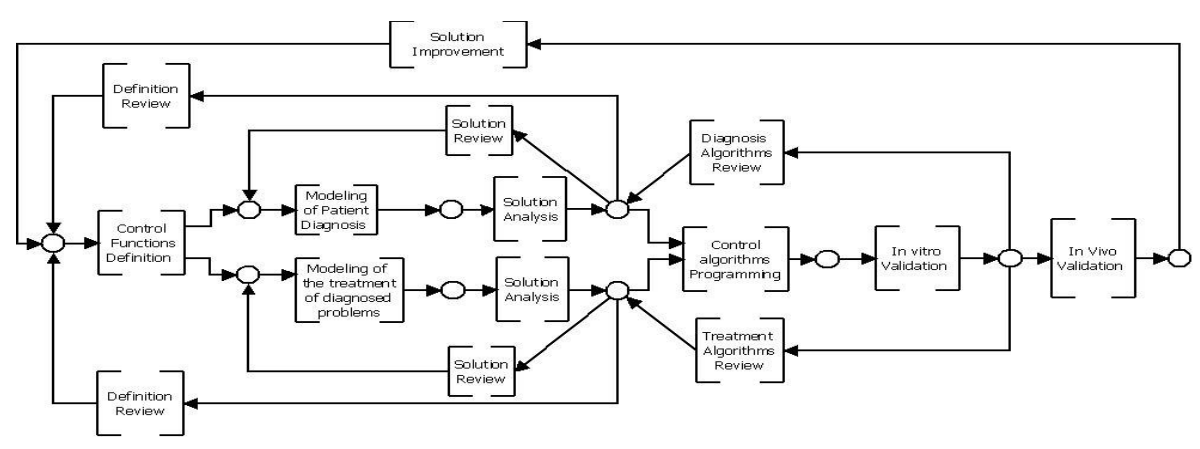

Fig. 3. Method for supervisory control system design applied to VAD;

The sequence proposed aims to organize the activities involved to develop control system design applied to VAD. So, these activities (represented by the elements of the activity model in the scheme proposed PFS) are presented briefly:

- Definition of control functions - A team of doctors and engineers defines the degree of autonomy of VAD control system. The team responsible control system development needs to select ideas that are possible to be implemented taking into account: sensors available, VAD performance characteristics and technological limitations. At this stage, a table with VAD control functions is specified.

- Modeling of Patient Diagnosis - Initially, diagnosis model involves development of a cause and effect matrix, which is basis for a BN. Then, this network can be converted into a HPN model.

- Modeling of treatment for diagnosed problems - From HAZOP (hazard and operability) [13] study the risk analysis report from VAD is obtained. Based on this information, we have the SIF, SIL and events (from sensors) and actions (to actuators) for each SIF. Next, diagnostic model and corresponding SIFs are modeled in HPN for control system design. 
- Solution Analysis - First, structural analysis of HPN model of diagnostic process is made. Next, it is checked if the HPN has no deadlock states (markings where no transition is enabled). For at, a simulator [14] can be used.

- Control algorithm programming - A Programmable Controller (PC) is an essential equipment for implementation of control systems. Consequently, standards have been set for this equipment allowing the reuse of existing software modules and ensuring high quality solutions, especially for conditions that require security methods for verification and validation. Thus, it is necessary to adopt following procedure: generation control program in programming language according to IEC 61131-3 [15], based on conversion of HPN models.

- In vitro validation for control algorithm - Control algorithms can be validated using mathematical model that simulates human cardiovascular system. The entire electrical equivalent of the model is shown in Figure 4. The electronic parameters are correlated to their mechanical parameters as follows: voltage (volt) is analogous to pressure $(\mathrm{mmHg})$, capacitance $(\mathrm{lF})$ to compliance $(\mathrm{ml} / \mathrm{Pa})$, resistance $(\mathrm{kX})$ to resistance $(1 \mathrm{~Pa} . \mathrm{s} / \mathrm{ml})$, and inductance $(1 \mathrm{H})$ to inertance $(1$ Pa.s2/ml) [16]. The elements of each artery including one or two resistor, an inducer and a capacitor. Figure 4(a) belongs to the arteries with the radius of less than $0.2 \mathrm{~cm}$ and figure $4(\mathrm{~b})$ belongs to the rest of the arteries. The architecture used to validate VAD control with cardiovascular system presented in Figure 1. Next step is prototype implementation that can be validated by a simulator of cardiovascular system. Currently, the Institute Dante Pazzanese of Cardiology (IDPC) has a programmable mechanical simulator that performs in vitro testing and is able to simulate real situations that can occur in patient's behavior [19].

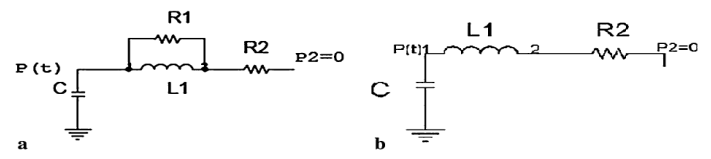

Fig. 4. (a) Electronic elements equivalents arteries with radius less $0.2 \mathrm{~cm}$ (b) others arteries.

- In vivo validation for control algorithm - After simulation and in vitro validation of control algorithm, VAD is ready for in vivo tests in calves [2].

Applying this set of procedures, is definition of a method for supervisory control system design is possible and capable to provide changes in VAD rotation speed, according to changes in cardiac frequency of patient, and can improve security and quality of life for patient who needs this type of device.

\section{Discussion of Results and Contributions}

The project of the control system of VAD according to the procedure previously considers:: (i) critical faults from HAZOP study for VAD, (ii) BN for diagnosis and decision, (iii) definition of Safety Instrumented Functions (SIF) using HPN and (iv) modeling of supervisory control system considering discrete and continuous 
variables of VAD. The result is the logical ordering of supervisory control system functions that are shown in Figure 3 based on PFS formalism (Figure 5). To implement this control system for VAD at IDPC is proposed the architecture according to Figure 6.

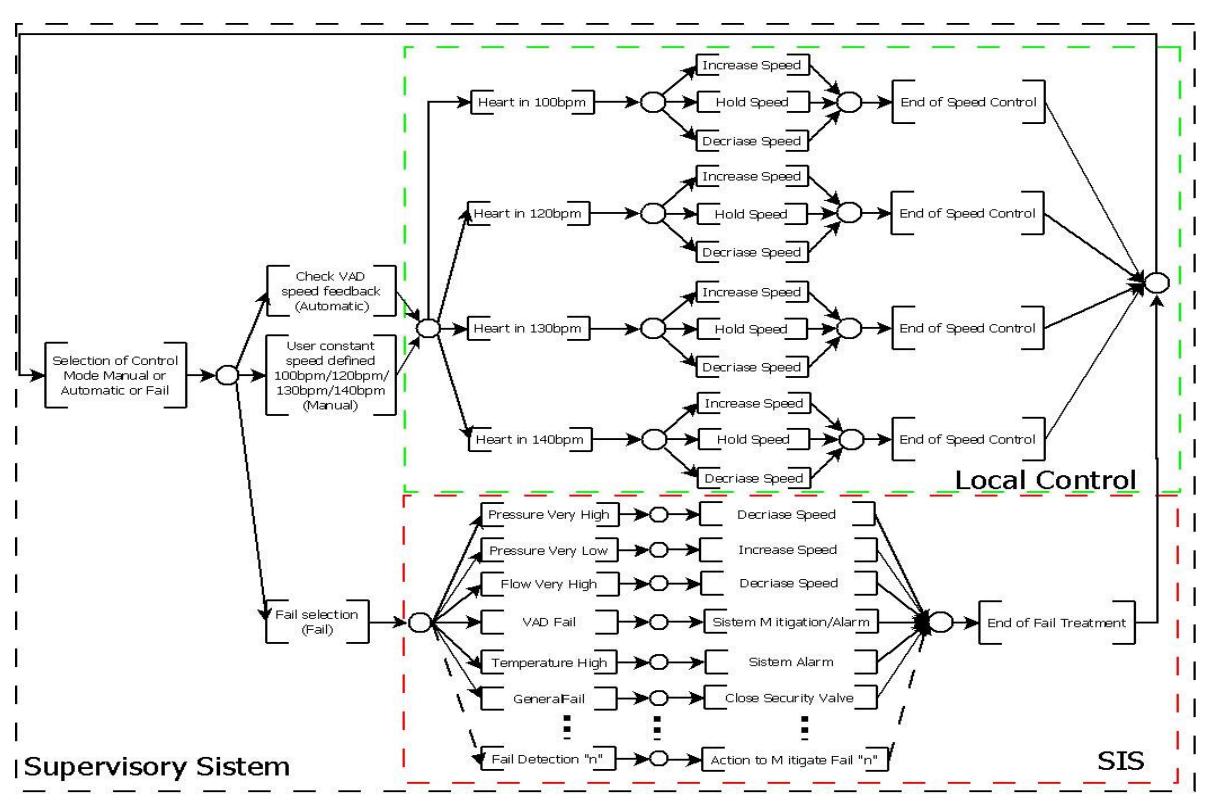

Fig. 5. PFS model to VAD supervisory control system.

According to this work proposal, each activity of model presented at PFS of Figure 5 is represented by a place on HPNs, and the marks can represent local and global states of VAD control system. Transitions are synchronized with human body reactions through adequate sensors. Oriented arcs can define the sequence for control functions processing. Therefore, we apply obtain VAD control algorithm based on proposed method in the proposal control architecture as show in Figure 6.

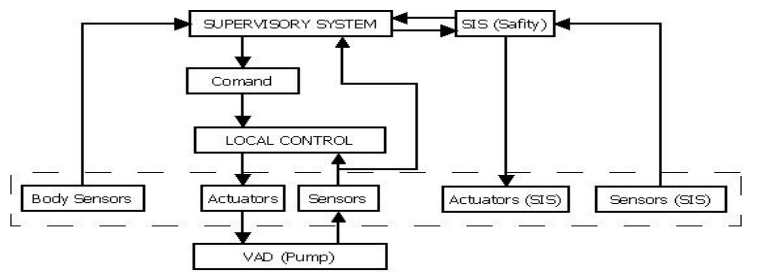

Fig. 6. Control architecture proposed.

Therefore, the control system proposed can improve VAD and can fit patient needs providing better patient quality of life and longer survival. Supervisory system can also assist in diagnosis and in interventions to maintain VAD functions. 


\section{Conclusion}

The VAD under development at IDPC presents difficulties concerning blood flow adjustment according to patient state: there is no device supervisory control that can adjust rotation speed based on patient needs and there is no treatment of VAD faults that can help patient's safety.

With a control system automatic and dynamic is possible that VAD adjust patient needs, providing a higher quality of life and enabling a patient survival. The supervisory system can also assist in diagnosis and possible interventions that doctors need to VAD control. Thus, VAD system may be adapted to the patient needs, keeping security and provides risks reduction. Therefore, VAD supervisory control system proposed offers advantages compared to currents VADs control systems.

This study contributes to have a customized VAD considering the patient's illness and different metabolism. Moreover, Safety Instrumented System concept is essential to provide risk reduction that might interfere in VAD functioning and in patient's life.

\subsection{Future works}

About the method to obtain VAD control architecture:

- Make research about a most efficient method for setting requirements to improve control system autonomy, concerning items complexity to determine the control and security system and to optimize process to obtain supervisory system.

About sensors and actuators used:

- Work to improve sensors to make them less invasive and allow to provide signals with higher quality and precision;

- Work to improve the dynamic pump features to improve system efficiency.

- Add block valves to VAD applying new technologies and using biomaterials.

Acknowledgments. We are grateful support of FAPESP and MEC/CAPES/PET.

\section{REFERENCES}

1. Wada, E. A. E. ; Andrade, A. J. P. ; Nicolosi, D. E. C. ; Bock, E. G. P. ; Fonseca, J. W. G. ; Leme, J. ; Dinkhuysen, J. J. ; Biscegli, J. F. . Review of the spiral pump performance test, during cerdiopulmonary bypass, in 43 patients. ASAIO Journal, v. 1, p. 1-2, 2005.

2. Andrade, A. J. P. ; Nicolosi, D. E. C. ; Lucchi, J. C. ; Biscegli, J. F. ; Arruda, A. C. F. ; Ohashi, Y. ; Muller, J. ; Tayama, E. ; Glueck, J. ; Nosé, Y. . Auxiliary total artificial heart: A compact electromechanical artificial heart working simultaneously with the natural heart. Artificial Organs , v. 23, p. 876-880, 1999.

3. Fonseca, J. W. G. ; Andrade, A. J. P. ; Bock, E. G. P. ; Leme, J. ; Dinkhuysen, J. J. ; Paulista, P. P. ; Manrique, R. ; Paulista, P. H. ; Valente, P. ; Nicolosi, D. E. C. ; Biscegli, J. F. . In vivo tests with the auxiliary Total Artificial Heart as a left ventricular assist device in calves. ASAIO Journal, v. 1, p. 1-5, 2005 
4. Ohashi, Y.; Andrade, A. J. P.; Muller, J.; Nosé, Y. . Control System Modification of an Electromechanical Pulsatile Total Artificial Heart. Artificial Organs, v. 21, n. 12, 1997.

5. Fonseca, J. W. G. ; Andrade, A. J. P. ; Nicolosi, D. E. C. ; Biscegli, J. F. ; Legendre, D. F. ; Bock, E. G. P. ; Lucchi, J. C. . A New Technique to Control Brushless Motor for Blood Pump Application. Artificial Organs, v. 32, 2008

6. Bock, E. G. P. ; Ribeiro, A. A. ; Silva, M. ; Antunes, P. I. T. C. ; Fonseca, J. W. G. ; Legendre, D. F. ; Leme, J. ; Arruda, A. C. F. ; Biscegli, J. F. ; Nicolosi, D. E. C. ; Andrade, A. J. P. . New Centrifugal Blood Pump With Dual Impeller and Double Pivot Bearing System: Wear Evaluation in Bearing System, Performance Tests, and Preliminary Hemolysis Tests. Artificial Organs, v. 32, 2008.

7. Villani, E.; Miyagi, P.E.; Valette, R. Landing system validation based on Petri nets and a hybrid approach. IEEE Transactions on Aerospace and Electronic Systems, Piscataway, NJ, v. 42, n. 3/4, p. 1420-1436, 2006.

8. Ho Y. Discrete Event Dynamic Systems : Analysing Complexity and Performance in the Modern World. In: IEEE Press, 1992.

9. Matsuno H, Tanaka Y, Aoshima H, Doi A, Matsui M, Miyano S: Biopathways representation and simulation on hybrid functional Petri net. In Silico Biol, 2003.

10.Squillante, R. et al. "Safety instrumented system designed based on Bayesian network and Petri net", 8th Intern. Conf. on Mathematical problems in Engineering, Aerospace and Sciences (ICNPAA), São José dos Campos, Brazil, 2010.

11.Pearl, J. Probabilistic Reasoning in Intelligent Systems: Networks of Plausible Inference.; San Mateo, CA: Morgan Kaufman Publishers.; 1988.

12.Cooper G.F., and Herskovits E., "A Bayesian method for the induction of probabilistic networks from data", Machine Learning, vol.9, pp. 309-347, 1992.

13.IEC, International Electrotechnical Commission, Functional Safety of Electrical / Electronic / Programmable Electronic Safety-related Systems (IEC 61508), Geneva, Switzerland, 1998.

14.Visial Object Net; Petri Net based Engineer Tool; version 2.7a; Copyright Dr. Rainer Drath; 2007. Available at http://www.paramsoft.de/

15.IEC, International Electrotechnical Commission, Programmable Controllers Part 3, Programming Languages, IEC1131-3 . Geneva: IEC, 1993. 207p.

16.Mona A., Mahdi N., Kamran H. Mathematical Modelling and Electrical Analog Equivalent of the Human Cardiovascular System, Cardiovasc Eng, 2010.

17.Reed T.R., Reed N.E., Fritzson P., Heart sound analysis for symptom detection and computer-aided diagnosis, Simulation Modelling Practice and Theory, 129-146, 2004.

18. Sainte M. J., Chapelle D., Cimrman R., Sorine M., Modeling and estimation of the cardiac electromechanical activity, Computers and Structures, 1743-1759, 2006.

19.Andrade, A. J. P. ; Filipini, C. L.; Lucchi, J. C.; Fonseca, J. W. G.; Nicolosi, D. E. C. . An electro-fluid-dynamic simulator for the cardiovascular system. Artificial Organs ,v.32, 2008.

20.Zickler,A, \& Mennicken, L.: Science for Sustainability: The Potential for GermanBrazilian Cooperation on sustainability-oriented Research and Innovation - Introduction. In: Proceedings of the 1st German-Brazilian Conference on Research for Sustainability, São Paulo, Brazil, 2009. 